\title{
Dal triangolo di Ippocrate al quadrato post-moderno: uno spazio di bioetica renale
}

\author{
Mario Timio $^{1,2}$, Francesca Timio ${ }^{1}$ \\ ${ }^{1}$ Dipartimento di Medicina Interna e Nefrologia e Dialisi, Ospedale di Foligno, Foligno (PG) \\ ${ }^{2}$ Direzione Scientifica Centro di Bioetica, Regione Umbria, Perugia
}

\begin{abstract}
From Hippocrates triangle to post-modern square: the new role of bioethics
The new era imposes modern strategies in the health care that change the Hippocrates triangle to the post-modern square. Then a new approach to bioethics rules is requested. This paper is focused on drugs production and distribution. With the assurance that ethical theories can solve moral quandaries. Egalitarianism defines health care as a right. This is a right of equal access to all that is available, which would include dialysis, transplantation and other advances in treating ESRD which may be developed. Including drugs stopping the decline of renal disease. But what does it happen when these drugs are too expensive? For the single and for the society? Guaranteeing everyone a right to treatment for ESRD, as is in EU, may in fact be so costly and deprive the populace of other basic needs, both medical and non-medical. As egalitarian point of view resonates with ethic tradition which eschews social worth judgment for allocation, we favor the use of expensive renal drugs, unless of proved efficiency. In this context we report the use of inhibitors of p-cresol, a strong nephrotoxic and cardiotoxic agent, and of drugs developed by precision medicine to treat progression of ESRD, such as tolvaptan in Polycystic Kidney Disease and sofosbuvir for HIV and its renal consequences.
\end{abstract}

Keywords: Drugs production, Hippocrates triangle, New ethical rules

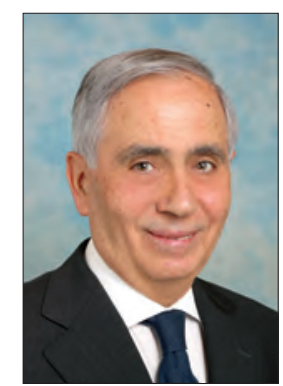

Dal triangolo di Ippocrate al quadrato post-moderno: nuovo ruolo della bioetica

Ippocrate immaginava il mondo della salute racchiuso in un triangolo ai cui angoli poneva tre M: Malattia, Malato, Medico. È un modello che è andato avanti per secoli nella storia della salute, fino a quando non ha fatto irruzione un'altra $M$ definibile "Mondo esterno" e il triangolo si è trasformato nella figura geometrica del quadrato. Nella quarta $\mathrm{M}$ entrano tante medicine, da quella tecnologica a quella diagnostica e curativa, da quella preventiva a quella narrativa, da quella di precisione a quella personalizzata, da quella economica a quella sociale, da quella organizzativa a quella sul territorio e da quella basata sulle evidenze a quella

Accepted: October 28, 2016

Published online: December 1, 2016

Indirizzo per la corrispondenza:

Prof. Mario Timio

Via XX Settembre 22

06100 Perugia

timma@libero.it riparativa. Soltanto per citarne alcune, nella consapevolezza che l'elenco si allunga sempre di più aggiungendo elementi che possono mettere in disparte la triade di Ippocrate. L'aggiunta di un quarto angolo suggerisce la debolezza dell'identificazione del ruolo del medico, sospeso tra l'approccio tecnologico e il recupero delle competenze antropologiche. II medesimo angolo mette in guardia dal considerare la malattia come parte emergente dell'iceberg clinico, dallo studiare e curare solo ciò che è palese e manifesto e dall'applicare una tecnologia antropologica che prescinda dall'unicità della persona e dalla sintesi tra soma e psiche e tra corpo e mente. Nel passaggio dalla figura geometrica del triangolo a quella rettangolare emerge la componente bioetica della medicina. Anzi, possiamo dire che la bioetica è la figlia maggiore della nuova medicina quadrangolare, che, in sintesi, pone l'uomo e la sua dignità all'incrocio dei quattro angoli. La bioetica si interessa, come abbiamo ripetutamente sostenuto e dimostrato anche in questa rubrica, dei problemi innescati proprio dall'aggiunta della quarta $\mathrm{M}$ con le sue varie e crescenti componenti, che entrano negli ambulatori, nei laboratori, nelle corsie e nei day-hospital, insomma in ogni letto dove ci sono un malato disteso e intorno un medico che tende a rialzarlo. Questi sa che dietro ogni malato c'è un progetto di vita che fa di un uomo "una persona morale unificata, cosciente" (1). E la finalità più diffusa di tale progetto di vita, secondo l'epistemologo Karl Popper, "è il compito personale di provvedere alla sopravvivenza sua e di quanti vivono con lui" (2). È il medico che entra nella casa e nella vita di tutti, un medico 
curante sempre disponibile, anche con le scoperte tecnologiche più recenti, poiché la sua professione non è semplicemente un mestiere, ma una vita. Sua e del paziente. II tutto secondo i canoni della moderna bioetica.

\section{Qualche applicazione bioetica in campo clinico}

Giovanni, anni 28, dipendente di un'impresa produttrice di cashmere. Da cinque anni è affetto da glomerulonefrite cronica, con lento ma graduale declino della funzionalità renale. Non accetta questa condizione; forse per questo è sempre più scontroso e taciturno. Sa che la dialisi (e il trapianto) è dietro l'angolo ed è perfettamente conscio che il suo progetto di vita è deviato, è modificato ed è distorto da eventi o casualità che lo agitano e mettono in forse il divenire dello stesso progetto. La nefropatia modifica ogni sua aspettativa poiché viene a infrangere temporaneamente o permanentemente il progetto. Parla e chiede poco, almeno fino a un certo punto della sua storia clinica. Ha la sensazione, e questo lo esterna successivamente, che i medici riescano scarsamente a penetrare nel labirinto della sua menomazione fisica e, ancora meno, nel groviglio delle sue sensazioni psicologiche a questa connesse. Li accusa di non piegarsi in ascolto, come lui vorrebbe, dei suoi problemi e di non far emergere le sue aspettative frustrate. Dalle poche parole che riesce a proferire traspare chiaramente il soffrire psicologico, più di quello fisico, della recisione della sua speranza progettuale. Poi, gradualmente, quanto più si avvicina la dialisi, inizia ad aprirsi con domande sempre più pressanti. A questo punto riemerge il triangolo di Ippocrate con le tre M: Malattia, Malato, Medico. Dice il padre della medicina: "Se il malato lascia che il medico combatta da solo contro il male o, anzi, gli si oppone prendendo le parti della malattia, accadrà che il medico sia vinto dalla malattia. Se, invece, l'ammalato gli si fa compagno contro di essa, sarà grande la speranza di vittoria". Ma Giovanni si fa "compagno" del medico solo perché vuole sapere se ci sono nuovi farmaci per arrestare la malattia o, meglio, per sapere se quello che ha letto sui media è vero o falso sulla presunta efficacia di alcune sostanze in grado di arrestare la progressione dell'insufficienza renale, liberando il paziente dall'incubo della dialisi. Così, un giorno si presenta con il foglio di un quotidiano in cui si annunciano nuovi alleati nella lotta contro la sua malattia. Uno di questi è una miscela di otto ceppi probiotici e di tre diverse fibre prebiotiche (Probinul 5), che, somministrata a pazienti con malattia renale cronica $(\mathrm{MRC})$, favorirebbe il rallentamento della progressione della malattia (3). È quello che Giovanni cerca e il medico ancora non gli ha somministrato. Perché? Per una questione di bioetica.

Intanto la miscela agisce sulla produzione intestinale (è assodata la stretta relazione tra rene e tratto gastrointestinale) di p-cresolo, una sostanza nefrotossica e cardiotossica, con la mediazione del riequilibrio del microbiota, comunemente chiamato "flora batterica". La terapia manca ancora di un anello importante, prima di essere somministrato liberamente, con o senza richiesta del malato: quello di trial randomizzati controllati, secondo i dettami della moderna sperimentazione. Uno di questi, denominato NATURE (Giovanni già ne conosceva l'esi- stenza), è in fase di avvio e prevede lo sviluppo di un integratore simbiotico, costituito da batteri probiotici, fibre e antiossidanti di derivazione mediterranea, da somministrare a pazienti con MRC. II gruppo di studio è quello del Prof. Gesualdo dell'Università di Bari, al quale Giovanni vorrebbe chiedere di essere inserito tra i nefropatici come oggetto della sperimentazione. Per accelerare la guarigione. Sempre sperando di non cadere nel gruppo di controllo con sostanze placebo e, quindi, inefficaci. Quindi, ancora problemi di bioetica. Ma ancora problemi di bioetica se consideriamo la tematica della medicina di precisione.

\section{La medicina di precisione}

"Ci sono due modi di stare al mondo, ha detto Einstein: possiamo vivere come se nulla sia un miracolo oppure scorgere un miracolo in ogni cosa" (4). E noi crediamo che la medicina di precisione, come emblema dell'angolo aggiuntivo del quadrilatero post-moderno, sia proprio un miracolo della medicina, almeno nel momento storico attuale. Cos'è la medicina di precisione? La medicina di precisione è una sfida nel mondo scientifico attuale. Essa interessa non solo medici, sperimentatori e programmatori, ma tutta la popolazione nella sua quotidianità (5). Da anni rappresenta lo sforzo di modellare la prevenzione e di modulare la diagnosi e la terapia della malattie sulle caratteristiche del singolo o di un gruppo di persone. Ambiente e stile di vita, in combinazione con la genetica, argomentava Umberto Veronesi, rendono ogni patologia diversa da un'altra, anche se porta lo stesso nome (6). Quindi, terapie personalizzate, o meglio, terapie non solo ripensate attorno all'ideale di una crescente precisione della diagnosi e della cura, ma anche al tipo di organizzazione dei servizi e dei sistemi sanitari nelle varie ramificazioni, con le quali si interseca l'intero edificio della medicina e della società. Quindi, si tratta di una frontiera molecolare e sociale nello stesso tempo. Coniugare i big data della biologia con i big data della società è il presupposto epistemologico della medicina di precisione. E del nostro futuro. C'è chi sostiene che la conoscenza in anticipo della risposta permetterebbe di mettere a punto regimi terapeutici adeguati. Per i nefrologi sarebbe già una conquista. Si pensi all'impatto personale e sociale se la medicina di precisione potesse essere applicata alle malattie che conducono alla MRC. Se pensiamo che oggi, in Italia, esistono due milioni di pazienti con MRC e 50000 nefropatici in dialisi e che ogni emodialisi costa circa 40000 euro per paziente per anno, la ricaduta economica della medicina di precisione è eclatante. Barak Obama ha annunciato un investimento di 215 milioni di dollari sulla precision medicine già dal 2015, per costruire una medicina che sia in tutti i sensi a misura d'uomo e di tutti gli uomini. Anche la Gran Bretagna ha investito una grossa somma per sequenziare, con il 100.000 Genomes Project, il genoma di 100.000 inglesi, entro il 2017, per aumentare le conoscenze delle malattie e per effettuare una conseguente terapia personalizzata. Ma quali saranno le persone ammesse al progetto e alle terapie conseguenti? Non è dato sapere. È auspicabile che non si ripeta ciò che è avvenuto nello stesso Paese all'inizio dell'era 
dialitica quando venivano ammessi al trattamento solo pazienti al di sotto di 60 anni, creando grossi problemi di bioetica.

\section{Farmaci troppo costosi: quando e a chi sono utili?}

Ma, ancora, problemi di bioetica emergono quando si tratta di investire nel campo della produzione di farmaci in un momento in cui questi sono molto efficaci, quindi curano meglio, ma a prezzi molto più alti. Un esempio eclatante è la scoperta e la commercializzazione di un nuovo farmaco contro il virus dell'epatite C: il sofosbuvir. Le guarigioni sono passate dal $60 \%$ al $95 \%$. È molto singolare la storia di questo farmaco, messo a punto da una piccola azienda, la Pharmasset, poi acquistato per 11 miliardi di dollari dal colosso Gilead Science, il quale ha guadagnato 16 miliardi di dollari per il primo anno di commercializzazione e 19 il secondo, avendo venduto il prodotto a peso d'oro. Dal momento che, in seguito, la Gilead Science ha seguito la filosofia di vendere ai paesi del Terzo Mondo il prodotto a prezzi bassi e iperbolici all'Occidente, l'Italia si è trovata inizialmente a pagare 45.000 euro per ogni trattamento, per poi passare a prezzi più bassi diluiti nel tempo a causa (o in virtù) della concorrenza a cifre più miti (7). Lo stesso discorso vale per l'HIV, oggi malattia cronicizzata che richiede, comunque, una terapia continua. Mutatis mutandis è quanto sta accadendo da oltre cinquanta anni per la MRC trattata con dialisi. Se ci fosse un farmaco commercializzato in grado di "guarire" o arrestare la progressione della nefropatia verso la cronicità , come sembrano disponibili alcuni prodotti della famiglia dei vaptani (tipo Tolvaptan) per la Malattia Renale Policistica, emerge il dilemma bioetico: "meglio spendere poco e a lungo per curare un paziente cronicizzato oppure molto e subito per un farmaco che si pensa capace di eradicare o rallentare la progressione del male?" È un dilemma drammatico, poiché non si può chiudere la porta all'innovazione e alla speranza dei malati, così come non si può mettere e repentaglio il budget del Servizio Sanitario Nazionale.

Per riprendere la tematica del sofosbuvir, è certo che, se dovessimo trattare tutti gli italiani con epatite $C$ (circa un milione) con tale farmaco, I'Italia andrebbe in bancarotta. E, quindi, addio a tanti altri trattamenti anche salvavita. Che fare, allora? II buon senso vorrebbe che, intanto, si sperimentassero nuovi farmaci e, poi, si vedrà. Ma chi sperimenta? 0 , meglio, chi investe? Oggi il fattore economico è l'elemento chiave della ricerca (8). Più si investe più cresce la possibilità di trovare le novità farmaceutiche. In realtà, come precisa Silvio Garattini, "le Big Pharma destinano alla ricerca soltanto il 7 per cento del fatturato: la gran parte dei fondi è assorbita dallo sviluppo". Chi indirizza i ricercatori? I numerosi ricercatori che orbitano nelle nostre Università o nei centri di sviluppo scientifico percepiscono che il tutto è regolato dal parametro del profitto. D'altra parte, è noto che portare un nuovo farmaco in commercio costa un miliardo di euro. Solo 5 su 10000 prodotti studiati raggiungono la sperimentazione clinica e appena uno entra in commercio. Grandi investimenti, quindi, senza garanzia di coprire le spese né di restare sul mercato. Sono poche le aziende farmaceutiche che hanno la stessa fortuna prima della Pharmasset e, poi, della Gilead Science. Un principio, comunque, vale per tutti: se i costi sono folli, le terapie sono inutili. Oggi la ricerca è prerogativa di piccole start-up biotecnologiche in cui gli sperimentatori possono far sentire la propria voce per approfondire progetti che devono finire sul letto del paziente. Per il bene comune di tutti. E per scelte bioetiche sostenibili.

\section{Conclusioni}

Nel passaggio dal quadrato di Ippocrate al rettangolo postmoderno emergono scenari di bioetica, legati al tipo di medicina che si vuole affrontare nel contesto della moderna organizzazione della società. A fronte di nuove opportunità scientifiche e mediche, si staglia un quadro di crisi dell'industria farmacologica che determina, da una parte, tempi lunghi (mediamente una decina di anni) tra la scoperta di un nuovo meccanismo patogenetico (gene o target molecolare) e la disponibilità del farmaco al letto del malato e, dall'altra, la produzione di prodotti a un costo così elevato da non permetterne l'applicazione su vasta scala per non rischiare il collasso di vari servizi sanitari. Ci sono, oggi, conoscenze scientifiche e mezzi adeguati per aggredire vecchie malattie e vincerle. La genetica, la disponibilità di modelli sperimentali nuovi e la possibilità di analizzare nel dettaglio i percorsi delle malattie stesse forniscono una nuova piattaforma di ricerca avanzata e di diffusione farmacologica, parallela a norme bioetiche che non possono essere mai disattese. Anche i pazienti con malattie renali vivono queste attese che la scienza pone sempre nelle loro speranze, come già avviene per alcune forme di MRC e di Malattia Renale Policistica. Di fronte a dilemmi bioetici di scelta vale sempre l'angosciante interrogativo: "Vale di più l'esistenza del singolo o l'esigenza di una gestione razionale"? (9). Perciò, davanti a noi, sta sempre una sfida etica che si fonda su una visione antropologica da cui non si può sfuggire.

\section{Disclosures}

Financial support: No financial support was received for this submission. Conflict of interest: The authors have no conflict of interest.

\section{Bibliografia}

1. Rawls J. A theory of justice. Harvard University Press, Cambridge. 1971:408.

2. Popper KR, Eccles JC. L'lo e il suo cervello. Trad. It. Vol. I, Armando Editore, Roma. 1981:180.

3. AAVV. Nuovi alleati nelle battaglia contro la MRC. BoxMedia, Milano. 2016:7.

4. Irvine B. Einstein e l'arte di andare in bicicletta. Centauria, Milano. 2016:10.

5. Pellicci PG. Medicina di precisione. Come ci cureremo in futuro. Atti: X Conferenza, The Future of Science, Venezia. 2015:18.

6. Veronesi U. idem: 34.

7. Desnoyer A, Pospai D, Lê MP, et al. Pharmacokinetics, safety of a full dose sofosbuvir-based regimen given daily in hemodialysis patients with chronic hepatitis C. J Hepatol. 2016; 65:40-7.

8. Gaviraghi G. Sienabiotech: una nuova sfida scientifica e industriale in Italia. Kèiron. 2002;11:98-109.

9. Churchill LR. Theory of justice in: Kjellstrand CM, Dossetor JB. Ethical problems in dialysis and transplantation. Kluwer Academic Publisher, Dordrecht, Boston, London. 1992:21-34. 